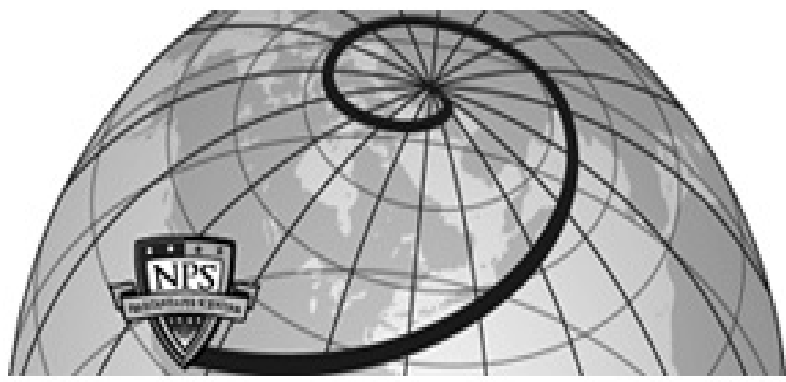

Calhoun: The NPS Institutional Archive DSpace Repository

\title{
High-Energy Particle Nomograph
}

Hoisington, David B.

https://hdl.handle.net/10945/41267

This publication is a work of the U.S. Government as defined in Title 17, United States Code, Section 101. Copyright protection is not available for this work in the United States.

Downloaded from NPS Archive: Calhoun

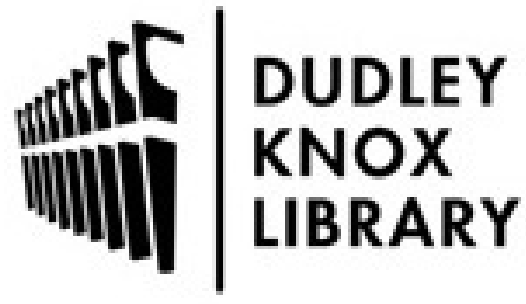

http://www.nps.edu/library
Calhoun is the Naval Postgraduate School's public access digital repository for research materials and institutional publications created by the NPS community. Calhoun is named for Professor of Mathematics Guy K. Calhoun, NPS's first appointed -- and published -- scholarly author.

Dudley Knox Library / Naval Postgraduate School 411 Dyer Road / 1 University Circle Monterey, California USA 93943 
Let $\vec{\Phi}_{0}$ have components $\phi_{i}>0$. Then, $\vec{S}_{1} \equiv \nu_{1}(1+\beta) \vec{\Phi}_{1}-$ $\beta \eta_{0} \vec{\Phi}_{0}$ has components:

$$
S_{i}=\nu_{1}(1+\beta) \psi_{i}-\beta \eta_{0} \phi_{i} .
$$

For $\beta \geq 0, S_{i}$ is positive $\langle=\rangle(1+\beta) / \beta>\left(1 / \nu_{1}\right)\left(\eta_{0} \phi_{i} / \psi_{i}\right)$, $\psi_{j}>0$ since the matrix $T$ has nonnegative elements. But $\eta_{0} \phi_{i} / \psi_{i} \leq \bar{\eta}_{1}$, so that $S_{j}$ is positive for all $j$ if $(1+\beta) / \beta>$ $\bar{\eta}_{1} / \nu_{1}$. This proves the first part. The second part follows from definition.

$Q E D$.

The above iteration sequence $\left\{\vec{\Phi}_{i}^{*}\right\}_{i=0}^{\infty}$ can be thought of as an extrapolation and renormalization performed on the sequence $\left\{\vec{\Phi}_{i}\right\}_{i=0}^{\infty}$. The norm-preserving feature of the definition of $\eta_{i} \vec{\Phi}_{i}^{*}$ is important when the last used fast group flux $\vec{\psi}_{0}$ is used as an initial approximation in the matrix equation:

$$
A_{2} \vec{\psi}=B_{2}\left(\eta_{i} \vec{\Phi}_{i}^{*}\right) .
$$

Thus, the norm of the vector of the right-hand side of the equation above is kept constant in the course of the iterations. It is fully expected that using the extrapolation and renormalization scheme with the proper $\beta$ 's will result in a reduction of effort in solving discrete matrix problems, the reductions being as much as 50 per cent.

\section{BIBLIOGRAPHY}

[1] Young, D. "Iterative Methods for Solving Partial Difference Equations of Elliptic Type," Transactions of the American Mathematical Society, Vol. 76 (1954), pp. 92-111.
[2] Birkhoff, G. and Varga, R. S. "Reactor Critically and Nonnegative Matrices," Bettis Atomic Power Division of Westinghouse, Pittsburgh, Pa., Report No. WPAD-166 (1957).

[3] Bellman, R., "On an Iterative Procedure for Obtaining the Perron Root of a Positive Matrix," Proceedings of the American Mathematical Society, Vol. 6 (1955), pp. 719-725.

[4] Frobenius, S-B. Preuss. Akad. Wiss. (1908), pp. 471-476.

[5] Stein, P. "A Note on the Bounds of the Real Parts of the Characteristic Roots of a Matrix," Journal of Research National Bureau of Standards, Vol. 48 (1952), pp. 106-110.

[6] Collatz, L. "Einschliessungssatz für die charakterisischen Zahlen von Matrizen," Math. Zeit., Vol. 48 (1942), pp. 221-226.

[7] Hildebrand, F. B. Methods of Applied Mathematics, New York, Prentice-Hall, Inc., 1952.

[8] Flanders, D. A. and Shortley, G. "Numerical Determination of Fundamental Modes, Journal of Applied Physics, Vol. 21 (1950), pp. 1326-1332.

[9] Aitken, A. C. "Studies in Practical Mathematics II. The Evaluation of the Latent Roots and Latent Vectors of a Matrix," Proceedings of the Royal Society of Edinburgh, Vol. 57 (1937), pp. 269-304.

[10] Shanks, D. "Non-linear Transformations of Divergent and Slowly Convergent Sequences," Journal of Mathematics and Physics, Vol. 34 (1955), pp. 1-42.

[11] Hestenes, M. R. "Iterative Computational Methods," Second Symposium on Applied Mathematics, Interscience (1955), pp. 85-95.

[12] Determination of Eigenvalues and Eigenvectors of Matrices, National Bureau of Standards Applied Mathematics Series No. 29 (1953), pp. 89-94.

[13] Bilodeau, G. G. "Extrapolation Techniques for Symmetric Matrices." Bettis Atomic Power Division of Westinghouse, Pittsburgh, Pa., Report No. WPAD-TM-52.

[14] Stark, R. H. "Rates of Convergence in Numerical Solution of the Diffusion Equation," Journal of Associated Computing Machinery, Vol. 3 (1956), pp. 29-40.

[15] Frankel, S. "Convergence Rates of Iterative Treatments of Partial Differential Equations," Mathematical Tables and Aids to Computation, Vol. 4 (1950), pp. 65-75.

\title{
High-Energy Particle Nomograph ${ }^{*}$
}

\author{
DAVID B. HOISINGTON $\dagger$
}

\begin{abstract}
Summary -A nomograph is presented which has proved to be useful in calculations related to the design of high-energy particle accelerators. Rest mass is given in the range from 0.0001 to 100 atomic mass units with the energy equivalent of this mass in electron volts. The final velocity and mass of the particle are given for a range of energies from 5000 to $10^{15}$ electron volts. The equation for particle velocity is given in the form
\end{abstract}

$$
v / c=\left[1-1 /\left(1+E / E_{0}\right)^{2}\right]^{1 / 2},
$$

where $E$ is the particle energy and $E_{0}$ is the energy equivalent of its rest mass. The frequency required for operation of a proton synchrotron is determined to illustrate the use of the nomograph.

$\mathrm{T}$ HE solution of many problems concerning highenergy particles requires a knowledge of the relationships between their energy, velocity, and mass. The design of particle accelerators and the measurement of particle energy are typical problems involving these relationships. Since the solution of the exact equations is

* Manuscript received by the PGNS, November 25, 1956.

$\dagger$ United States Naval Postgraduate School, Monterey, Calif. somewhat laborious, a nomograph (Fig. 1) opposite, has been constructed to facilitate the calculations where two-place accuracy is sufficient.

There are three axes on the nomograph. The first gives the rest mass of the particle, $m_{0}$, in atomic mass units (amu) as well as the energy equivalent of this mass, $E_{0}$, in electron volts. The second axis gives the kinetic energy of the particle, $E$, in electron volts. The third axis gives the velocity of the particle, plotted as $(1-v / c)$, and the ratio of its actual to rest mass, $m / m_{0}$. Any straight line intersecting the three axes shows the relationship between these quantities. Approximate equations are given in the Appendix for velocities lower and higher than those covered by the nomograph.

The frequency range of the accelerating voltage in a proton synchrotron will be determined to illustrate the use of the nomograph. Assume that the protons are injected with an initial energy of $4 \mathrm{mev}$ and accelerated to an energy of $2.3 \mathrm{bev}$ in an accelerating tube having a 

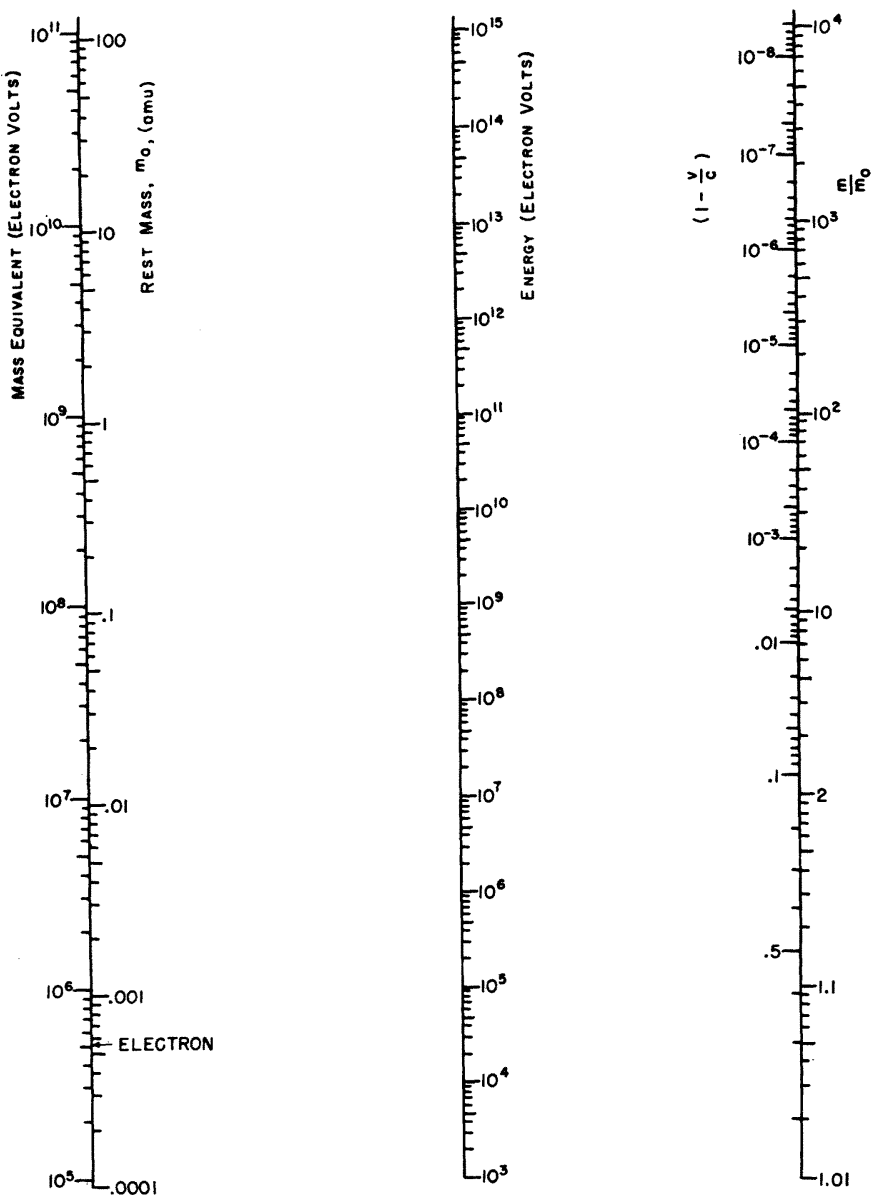

Fig. 1-Nomograph of mass and velocity of high-energy particles.

circumference of 68 meters. Reference to Fig. 1 shows that the final velocity of the protons is 0.958 cycles. The frequency at which the particles finally rotate is, therefore, $\left(0.958 \times 3 \times 10^{8}\right.$ meters $) /(68$ meters $)=4.2 \times 10^{6} \mathrm{cps}$. The energy of the injected particles is too low for their velocity to be given by the nomograph. From the approximate relationship given in the Appendix for lowenergy particles, $(v / c) \approx\left(2 E / E_{0}\right)^{1 / 2}=(2 \times 4 / 938)^{1 / 2}=$ 0.0924 . The corresponding rotational frequency is $\left(0.0924 \times 3 \times 10^{8}\right.$ meters $) /(68$ meters $)=0.41 \times 10^{6} \mathrm{cps}$.

An electron synchrotron is planned by M.I.T. and Harvard to accelerate electrons to an energy of 6 bev. Reference to the nomograph shows that the electrons will emerge from this machine with a mass 13,000 times their rest mass, and at a velocity within $4 \times 10^{-7}$ per cent of the velocity of light.

\section{Appendix}

Equations for Mass and Velocity

$$
\begin{gathered}
\frac{m}{m_{0}}=1+\frac{E}{E_{0}} \\
\frac{v}{c}=\sqrt{1-\frac{1}{\left(1+E / E_{0}\right)^{2}}}
\end{gathered}
$$

For $E / E_{0} \ll 1$ (Error $<2$ per cent if $E / E_{0}<0.025$, $v / c<0.22)$

$$
\frac{v}{c} \approx \sqrt{\frac{2 E}{E_{0}}}
$$

For $E / E_{0} \gg 1$ (Error $<2$ per cent if $E / E_{0}>3$, $(1-v / c)<0.03)$

$$
\left(1-\frac{v}{c}\right) \approx \frac{1}{2\left(1+E / E_{0}\right)^{2}}
$$

Energy Equivalents of Certain Particles

\begin{tabular}{lc} 
Particle & $E_{0}(\mathrm{mev})$ \\
\hline Electron & 0.51098 \\
Proton & 938.23 \\
Neutron & 939.52 \\
Deuteron & 1875.5 \\
Alpha & 3727.2
\end{tabular}

\title{
A Portable Scintillation Alpha Survey Instrument
}

\author{
W. G. SPEAR $\dagger$
}

\begin{abstract}
Summary - A scintillation alpha counter utilizing a zinc sulfide fluor, a multiplier phototube, and a two-stage vacuum tube amplifier has been developed. A neon-bulb oscillator high-voltage supply is used to supply 900 volts to the multiplier phototube, and headphones are used to obtain an aural indication of counting.

The average geometry or counting efficiency over the 7.43-inch ${ }^{2}$ probe area is approximately 13 per cent with variations from a maximum counting efficiency at probe center of 18 to 22 per cent
\end{abstract}

\footnotetext{
* Manuscript received by the PGNS, December 8, 1956.

$\dagger$ HAPQ Labs. General Electric Co., Richland, Wash.
}

down to approximately 7 to 8 per cent at the probe edges. The background counting rate is less than one count per minute, and the battery life is approximately 150 hours of continuous operation for the $3-1 / 2$ pound instrument.

\section{INTRODUCTION}

TCINTILLATION alpha counters are preferable, in 7 general, to air-proportional counters because they are inherently nonmicrophonic, little affected by humidity and temperature variations, and can be made to 